\title{
Organized Atrial Tachycardias after Atrial Fibrillation Ablation
}

\author{
Sergio Castrejón-Castrejón, ${ }^{1}$ Marta Ortega, ${ }^{1,2}$ Armando Pérez-Silva, ${ }^{1}$ David Doiny, ${ }^{1}$

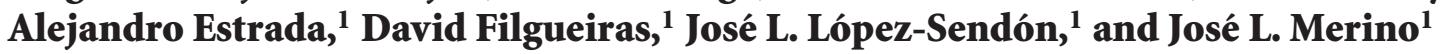 \\ ${ }^{1}$ Robotic Cardiac Electrophysiology Unit, Department of Cardiology, University Hospital La Paz, Paseo de la castellana, No 261, \\ 28046 Madrid, Spain \\ ${ }^{2}$ Department of Pediatric Cardiology, University Hospital La Paz, Paseo de la castellana, No 261, 28046 Madrid, Spain
}

Correspondence should be addressed to José L. Merino, jlmerino@secardiologia.es

Received 15 November 2010; Revised 17 April 2011; Accepted 17 May 2011

Academic Editor: Miguel A. Arias

Copyright (C) 2011 Sergio Castrejón-Castrejón et al. This is an open access article distributed under the Creative Commons Attribution License, which permits unrestricted use, distribution, and reproduction in any medium, provided the original work is properly cited.

\begin{abstract}
The efficacy of catheter-based ablation techniques to treat atrial fibrillation is limited not only by recurrences of this arrhythmia but also, and not less importantly, by new-onset organized atrial tachycardias. The incidence of such tachycardias depends on the type and duration of the baseline atrial fibrillation and specially on the ablation technique which was used during the index procedure. It has been repeatedly reported that the more extensive the left atrial surface ablated, the higher the incidence of organized atrial tachycardias. The exact origin of the pathologic substrate of these trachycardias is not fully understood and may result from the interaction between preexistent regions with abnormal electrical properties and the new ones resultant from radiofrequency delivery. From a clinical point of view these atrial tachycardias tend to remit after a variable time but in some cases are responsible for significant symptoms. A precise knowledge of the most frequent types of these arrhythmias, of their mechanisms and components is necessary for a thorough electrophysiologic characterization if a new ablation procedure is required.
\end{abstract}

\section{Introduction}

Organized atrial tachycardias (AT) are a common problem after atrial fibrillation (AF) ablation (post-AF ablation ATPAFAT). Since the first isolated case reports [1-5], several mechanisms [6] and different times of onset following the index procedure have been reported. A new ablation procedure often solves this arrhythmic problem [5, 7-14]. Nevertheless, this rhythm disorder merits a special attention for different reasons: (1) it has a high incidence and is often very symptomatic, (2) the complexity of the atrial arrhythmogenic substrate, which may be responsible for the frequent concurrence of several types of AT mechanisms in the same patient, (3) the variety of mapping and ablation approaches which have been reported and (4) the fact that PAFATs mechanisms may be linked to the mechanisms responsible for AF maintenance.

This paper reviews the incidence, clinical presentation, mechanisms, electrophysiological characterization and ablation of PAFAT. Finally, a brief review of organized ATs presenting during the $\mathrm{AF}$ ablation procedure is also provided.

\section{Incidence}

The real incidence of PAFAT cannot be easily extracted from published series because most of them either focused just on left [15-17] or macroreentrant (MR) AT [18, 19], or did not report the incidence of cavotricuspid isthmusdependent (CTI) atrial flutter, which is responsible for 7$10 \%$ [20-22] of all PAFATs. This latter figure is even higher in patients with previous cardiac surgery [23]. In addition, the reported incidence of PAFAT is probably underestimated in most of these series because only symptomatic patients were referred for a new ablation procedure $[21,24]$. All these reasons explain in part that the reported incidence of sustained PAFAT varies widely. However, differences in PAFAT incidence mainly depend on the following two factors: the predominant type of AF before the index procedure and the approach $[8,12,13]$ used for AF ablation.

The incidence of PAFAT ranges between 4.7 and $31 \%$ and is usually higher after circumferential pulmonary veins (PV) ablation using wide-area circular lesions around ipsilateral PVs (circumferential pulmonary vein ablation-CPVA) [25] 
or when additional ablation lines are incorporated in the procedure than with other ablation approaches [15-18, 20, $21,24,26,27]$. This group of patients whose AF was treated with CPVA is by far the one in which PAFATs have been most extensively studied. However, several variants of the original CPVA technique have been reported in recent years. Abatement of PV electrograms within the encircled area was the main endpoint of the original series and PV electrical isolation (PVI) has been required only in the most recent ones [26-28]. Despite this evolution [29], the PAFAT incidence apparently has remained apparently unchanged. The number and location of additional ablation lines had been also heterogeneus among the different reported series but it seems that additional lines may have a greater impact on PAFAT incidence than on AF recurrences. Pappone et al. [25] and Anousheh et al. [27] found that by adding roof, posterior, or mitral isthmus ablation lines the development of new-onset macroreentrant AT was reduced in comparison to CPVA alone, as far as conduction block across these lines was achieved.

The incidence of AT is much lower after less extensive AF ablation approaches such as segmental ostial PVI [1, 30, 31] or circumferential antral PVI [32], especially when no additional ablation lines are used or only the electrically active PVs are targeted [23, 24, 33-39]. The incidence of AT with these latter approaches ranges between $2 \%$ and $7.7 \%$. The only discrepant incidence value with this approach is the $29 \%$ found by Ouyang et al. [38] but that one could have been related to the modification of the standard PV isolation technique which these investigators used. The lower incidence of PAFAT found following segmental ostial PVI could be partially related to the smaller proportion of patients enrolled with persistent/chronic AF enrolled in these studies, as opposed to the studies in which CPVA was employed. Patients with long-standing persistent/chronic AF have both more electrical and anatomical atrial remodeling $[40,41]$ and low-voltage and scar areas [34] than patients with paroxysmal AF. These scar areas have been associated with MR circuits and may configure the substrate for organized AT [13, 34, 35]. This hypothesis was congruent with a ninefold higher AT incidence in patients with persistent forms of AF reported by Porter et al. (2,4\% versus 20\%) [42]. Actually, this is not the case because the proportion of patients with persistent/chronic AF in the studies in which CPVA plus lines was used reached 17\%-36,5\% [15$18,20,21,24,26,27]$, a percentage similar to that reported in the series in which segmental or circumferential antral PV isolation was performed (8\%-43\%) [23, 24, 33-39]. As a consequence, factors directly linked to the ablation technique in itself seem to determine the PAFAT incidence, although the scarce studies that have compared both techniques have yielded conflictive results $[43,44]$.

Finally, there are some other strategies globally characterized by an extensive atrial ablation which aims at terminating $\mathrm{AF}$, rendering it noninducible or at least transforming it into an organized AT amenable to mapping: complex fractionated atrial electrograms (CFAEs) ablation [45], alone or as an adjunct to PVI isolation, and stepwise approaches comprising sequential addition of conventional techniques
[46]. The incidence of PAFAT in patients initially treated with CFAE ablation alone or combined with PVI [45, $47-53$ ] is $7,6 \%-24 \%$, but Nademanee et al. published a remarkable study with 674 patients in which a low incidence of right atrial flutter $(2,4 \%)$ and no cases left AT [52] were reported. Most of the patients included in these series suffered persistent, permanent, or chronic AF (33\%-100\%). On the other hand, a number of papers have reported the incidence of AT after stepwise AF ablation or addressed specifically AT appearing after these approaches [46, 53-59]; the incidence of PAFAT in this context oscillates between $23 \%-44 \%$. Stepwise techniques are resorted to for longlasting persistent forms of AF almost exclusively (23\%-100\% of patients in these series).

\section{Clinical Aspects}

From a clinical point of view, PAFATs are characterized by: early onset after the index procedure, multiplicity of arrhythmia types in the same patient (not always recognizable on the surface ECG), frequent and important symptoms refractory to management with rate-controlling drugs, limited amenability with antiarrhythmic drugs, high recurrence rate after cardioversion, and to sum up, very frequent requirement of at least one subsequent ablation procedure to cure them.

3.1. AT Time of Onset after AF Ablation. Chang et al. [28] demonstrated that multiple ATs can be induced in 16,3\% of patients immediately after circumferential PVI isolation, and this proportion rises (38\%) when a more extensive ablation is used [19]. In other cases, an organized AT of a "totally" different nature such as typical CTI-dependent flutter appears during AF ablation [60]. These findings suggest that the substrate capable of maintaining organized ATs is already present at this stage. It may explain why the onset of AT occurs relatively early in the follow-up after AF ablation, with an average time of onset between 2, 7-13 weeks [15-19, 55]. Ouyang et al. [38] and Themistoklakis et al. [35] reported that more than $80 \%$ of the ATs they studied had appeared during the first 2 and 4 weeks after the AF ablation procedure, respectively.

3.2. Clinical Course. Chugh et al. [18] proposed to reserve a new ablation for patients in whom the AT eventually persists symptomatic after a prudential observation period, because according to their reported experience, up to a third of patients presented complete resolutions of their ATs (spontaneously or after electrical cardioversion) and an additional $4.7 \%$ achieved good clinical control under pharmacologic treatment alone. This cautious proceed is further guaranteed because in other studies, with different AF ablation techniques, $45-64 \%$ of patients remained free of AT recurrences after a few months had elapsed $[15,17,19$, 45].

Although PAFATs are most commonly persistent $(78 \%$ [59]-92\% [56]) instead of paroxysmal, they tend to cause important symptoms (even syncope in $28 \%$ of patients 
studied by Pappone et al. [17]). Commonly, PAFAT are more problematic for patients than the original AF, specially because organized atrial arrhythmias are usually associated to faster ventricular response thus requiring electrical cardioversion more frequently $[15,19,20]$. In addition, these PAFATs respond poorly to antiarrthythmic drugs (only $4.7 \%$ [22]-18\% [20] of patients on antiarrhytmics are oligosymptomatic enough as to reject a new ablation procedure), and in some cases interruption of antiarrhythmics could be beneficial to avoid recurrences [33].

3.3. Multiple Different Arrhythmias in the Same Patient. This is one of the most prominent features of PAFAT. Haïssaguerre et al. [56] and Deisenhofer et al. [15] described 70-81\% of their patients to have more than one arrhythmia mechanism during the electrophysiologic study. Other authors $[15,19$, $57,58,61]$ have reported that the average number of different AT mechanisms per patient varies between $1.8 \pm 1.2$ and 3.4 \pm 2.4 and that, in subsequent recurrences, the clinical AT is different to the original one in $79 \%$ of cases [21].

3.4. Predictive Factors of AT Occurrence. Several circumstances have been postulated to contribute to a more elevated risk of PAFAT: extensive ablation [21, 24, 26], incompletely ablated areas $[15,27,62]$, PV reconnection $[34,38]$, early debut of atrial arrhythmias post AF ablation [18, 21, 35, 63], and previous long-lasting $\operatorname{AF}[17,35]$. Extensive ablation may increase the incidence of AT both directly, creating conduction barriers that eventually would define and stabilize a reentry circuit spatially separated from the ablated area [21, 64], and indirectly, enhancing the probability of conduction gaps [17], sometimes with complex tridimensional structure [65].

\section{Types of PAFAT}

\subsection{Macroreentrant Circuits after AF Ablation (Figure 1)}

4.1.1. Frequency and Types. Macroreentrant (MR) ATs are considered the most frequent arrhythmias after AF ablation, comprising $57 \%-91 \%$ of the total. Nevertheless, some relevant studies have made clear that it may not always be so. Haïssagerre et al. [56] and Deisenhoffer et al. [15] reported that the so-called small-loop or localized reentry could be as frequent a mechanism as MR. Shah et al. [37], Ouyang et al. [38] and Gerstenfeld et al. [33] published data according to which focal or small-loop reentry was the most frequent mechanism (77\%, 88\%, and 100\%, resp.). These striking differences could reflect not only, in the first instance, a renewed interest in a more detailed characterization of the frequently neglected "focal" atrial tachycardias [6668], but also the possibility that more limited ablation strategies such as those used in these studies (PV segmental or circumferential isolation alone $[33,37,38]$ ) are not so liable to cause MR tachycardias. Perimitral atrial flutter is the most common type of MR circuit arising after AF ablation $(39 \%-61 \%)$, closely followed by roof-related or peri-PV tachycardias (9\%-61\%), typical right atrial flutter

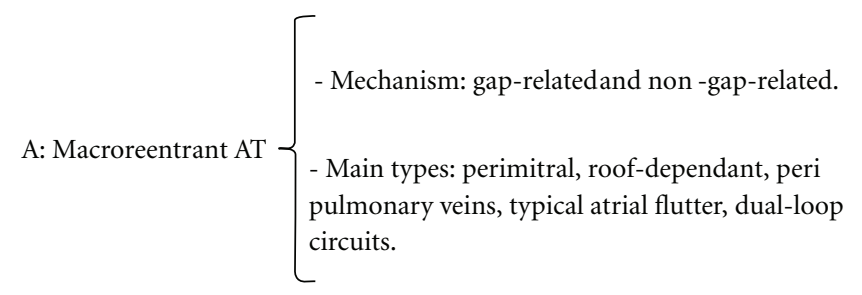

B: Small-loop/localized
reentrant AT $\left\{\begin{array}{l}\text { - Associated to conduction gaps in ablation lines. } \\ \text { - Associated to very slow conducting areas. }\end{array}\right.$

C: Pulmonary vein AT $\longrightarrow$ Reconnected pulmonary veins.

D: Genuine focal AT $\longrightarrow$ Triggered activity or abnormal automaticity.

Figure 1

(15\%, although in some case series this was the most frequent arrhythmia $[45,52])$, and other circuits involving the coronary sinus $(5 \%-7 \%)$, the interatrial septum $(10 \%-$ $18 \%)$, or the anterior atrial wall [64] (3\%) [15-21, 33, 37$39,45,52,54,56,57,62,69]$. It is noteworthy that complex dual-loop circuits are not rare $(22 \%-55 \%)[16,21]$.

4.1.2. Identification and Mapping of MR Circuits. This mechanism is suspected when the activation sequence map yields results congruent with a sequentially continuous electrical activity accounting for at least $80 \%-90 \%$ of the tachycardia cycle length (CL). Activation sequence maps are usually obtained with tridimensional (3D) electroanatomic mapping systems in which every local activation time is represented as a color of a continuous spectrum, thus creating a visual representation of the sequence of activation. If the diameter of the circuit is $\geq 3 \mathrm{~cm}$, the earliest activated area is close to the latest one (the typical "early meets late" or "head meets tail" aspect), and if the range of local activation times encompasses most of the CL, macroreentry is probable. The boundaries delimiting the circuit (such as sites harboring double potentials separated $\geq 50 \mathrm{~ms}$, low-voltage areas characterized by voltages $\leq 0,05-1 \mathrm{mV}$, anatomical obstacles) are commonly included in the activation map representation in order to depict the course of the activation front with more anatomical accuracy. This method of creating an activation sequence map was the most frequently used in the studies previously cited. In spite of its widespread acceptation, activation maps can be sometimes misleading $[56,70]$ and lead to equivocal results. Typically, when the entire MR circuit is not accessible or some parts of the activation sequence cannot be registered with certainty [36, 71], the activation map of a MR circuit can be misinterpreted as an apparent centrifugal activation of the atria with a presumptive, but false, focal origin [36]. The limitations 
of the activation mapping are posed by a nonsystematic collection of points during which critical areas of the circuit [72] may be missed, the intrinsic difficulty of assigning an objectively exact activation time to a multicomponent or low-voltage fractionated electrogram, and the presence of multiple areas of slow conduction or conduction block resulting in an abnormally prolonged time to complete LA activation [73] in such a way that some atrial areas are activated very late. Some modifications of the conventional point-by-point construction of activation maps have been attempted in order to simplify the approach and gain accuracy. For example, the use of multielectrode catheters [72] or the elegant refinement of this technique reported by De Ponti et al. [74].

In addition, the documentation of a sequential atrial activation through the entire AT cycle length is insufficient to establish the existence of an atrial MR arrhytmia: the only unequivocal proof is the demonstration of fusion during entrainment or, what is even more compelling, return cycles after entrainment matching the tachycardia CL from at least two distant sites. In this respect, entrainment mapping with fusion affords the ultimate evidence of a reentrant mechanism irrespective of the "focal" appearance of the activation map $[33,36,39,71]$. A detailed analysis of entrainment maps has not been carried out in most studies: in some cases entrainment techniques have been used [16, $19,20,28,34,75]$ only exceptionally, and in other cases entrainment has been used in a limited way just to confirm that certain sites of interest (particularly putative isthmuses candidate for ablation) belong to the circuit $[17,21,22$, $58,62,72]$. On the other hand, entrainment mapping has been used by some investigators in a more comprehensive manner to determine the true spatial location of the circuit $[15,36,57,64]$. Some authors have manifested a certain reluctance against an exhaustive use of entrainment mapping [76] not only because of its limitations (it is not always possible to demonstrate fusion, specially when reentrant circuits are small [37]) but also because there exist a potential risk of terminating the AT into sinus rhythm, a different AT or AF.

\subsection{Focal Mechanisms (Figure 1)}

4.2.1. Definition and Types. The identification of a focal AT [6] is based on two criteria: (a) centrifugal spread of the activation front in all directions from the site of earliest atrial activation [75], (b) range of activation duration less than the AT CL $[16,17]$, or in other words, sequential electrical activity accounting for less than $80 \%-90 \%$ of the CL [21, 22]. However, it is sometimes very difficult to discriminate between reentrant and focal mechanisms $[36,56]$, specially in a previously ablated atria. Due to this difficulty, a number of subordinate criteria have been proposed to suspect a focal origin $[6,33,53,57,68]$ : (a) CL variations $>10 \%$, (b) discrete P-waves with clear isoelectric intervals between them, (c) inconsistent return cycles after entrainment pacing from several locations, (d) identical $\mathrm{P}$ wave and atrial activation sequence during pacing at the focus site and during the clinical AT, and (e) QS wave on the monopolar lead located on the origin of the AT. It must be noted that a focal origin of the electrical activation does not necessarily involve a "genuine" focal mechanism, such as enhanced automaticity or triggered activity. In this regard, it might be useful to remember that any confusion of terminology must be avoided: the term "focal AT" refers primarily to a pattern of concentric activation from a focus/source [6], the mechanism of which could indistinctly consist on (micro) reentry, abnormal automaticity, or triggered activity. In consequence, the use of the term "focal" as a synonym for automatic or triggered mechanism is confuse [6].

A particular subgroup of focal ATs [77] are characterized by (a) adenosine insensitivity (they present neither termination nor transient suppression), (b) low-amplitude potentials at the focus site, (c) long-duration electrograms (spanning a great portion of the cycle length) at the origin site, and (d) a response to overdrive pacing consistent with entrainment. All these features, considered together, strongly advocate reentry as the underlying mechanism. Sanders et al. [68] studied 27 tachycardias with a catheter specially designed for high-density mapping, establishing a localized reentry mechanism in 8 of them. The most relevant finding of this approach was the indisputable demonstration of sequential electrical activity during $95.2 \pm 4.5 \%$ of the CL, together with prolonged fractionated electrograms at the sites of origin. Takahashi et al. [55] achieved similar results using conventional activation and entrainment mapping, but they also measured the size of the circuit (always $<2 \mathrm{~cm}$ ), the width of the isthmus (variable between $<5$ and $10 \mathrm{~mm}$ ) and confirmed the spatial relationship of these small-loop reentry circuits to previously ablated areas. The close relationship between low-voltage zones (LVZs) or scar areas and the site of origin of the focal AT was reassured by Higa et al. [67] using noncontact mapping. Surrounding LVZs can sometimes configure a preferential exit channel from the AT focus [78]. These microreentry or small-loop reentry circuits fairly explain why Mohamed et al. [79] demonstrated that the closer to the AT focus the entrainment site is located, the shorter is the return cycle minus CL difference, a type of response that suggest a reentrant circuit when can be obtained consistently [80]. Finally, such sites harboring localized reentrant circuits have been directly proved or strongly proposed to be involved in the maintenance of AF $[81,82]$.

Deisenhofer et al. [15] have differentiated two types of small-loop reentry circuits $(<3 \mathrm{~cm})$ on the basis of a very reasonable argumentation: (a) small-loop reentry circuits related to gaps on previous ablation lines and (b) small-loop reentry circuits related to areas with markedly slow conduction, generally located in close proximity to previously ablated areas but not related to conduction gaps. Schematic small-loop reentry circuits resultant from the modification of a previous arrhythmogenic substrate by radiofrequency lesions were hypothesized by Merino in 2006 [73].

The other two focal mechanisms (automaticity and triggered activity) have not been so well defined in patients presenting PAFAT and would remain an exclusion diagnosis 
when MR or localized reentrant circuits could not be confirmed. Their typical responses to adenosine and overdrive pacing along with their typical clinical presentation as repetitive burst of tachycardia can serve as clues to suspect the diagnosis $[77,83]$.

4.2.2. Frequency and Preferential Locations. In general, focal ATs constitute a minoritary group. For example, Deisenhofer et al. [15] did not report any focal ATs in their series, but it must be noted that $31 \%$ of the PAFATs they studied were too unstable for complete characterization. Gerstenfeld et al. [33], on the other hand, initially attributed a focal mechanism to all PAFATs they found after segmentary PVI. This assumption was somewhat doubtful because all these tachycardias manifested a fused intracardiac activation sequence during entrainment. On the basis of entrainment maneuvers the same authors published a second report [39] in which only one of five PAFATs had a presumable pure focal mechanism and the other four cases were small-loop reentries. These results underscore the capital importance of the differentiation between a focal activation pattern and a true focal mechanism. The first one habitually results from small-loop reentries or conduction barriers which can mask a MR mechanism [34, 73].

Rostock et al. [57], Chae et al. [21], and Mesas et al. [16] published a prevalence of focal AT which varies between 12$28 \%$ of the total PAFATs. The immense majority of these focal PAFATs were found near the PV antra in close relationship to previously ablated areas (41-100\%) [16, 21, 57]. Apart from this preferential location, PAFATs with a confirmed or putative focal mechanism have been described as being scattered in many other places such as the coronary sinus (23\%), interatrial septum (11-12\%), LA roof and LA inferior wall $[21,57]$.

\section{Electrophysiologic Characterization of PAFAT}

A precise knowledge of the arrhythmogenic substrate as well as a great familiarity with the electrophysiologic properties of AT circuits is essential to identify and ablate their critical components.

5.1. Conduction Gaps. Conduction gaps are simply narrow parcels of surviving cardiac tissue still capable of effective electrical conduction which connect two zones otherwise separated by an interposed area of nonconducting tissue. This area of conduction block generally consists on scar tissue caused by radiofrequency or other modalities of energy delivered for ablation. Therefore, a gap is always a portion of cardiac muscle which has been either reversibly damaged by the ablation catheter, independently of the nature and intensity of the lesion (inflammation, edema, alteration of membrane properties [84]) or not damaged at all. Obviously, gaps can be already present immediately after the AF ablation procedure. These residual gaps are typical in atrial regions which are hardly accessible to radiofrequency energy for whatever reason: catheter instability, edema formation, tissue thickness that prevents the transmurality of the lesions, or tricky anatomical areas such as the ridge between the left upper pulmonary vein and the left atrial appendage $[17,85$, 86]. More frequently, however, conduction gaps appear later as true "reconnections" [87] of the previously ablated areas.

5.1.1. Relationship between Gaps and PAFAT. Despite multiple targeted radiofrequency applications during an AF ablation procedure, multiple or single gaps can persist in a significant number of patients (5-19\%) [17, 88, 89]. These incomplete lines have been proved to be a strong promoting factor of AT $[17,27,43]$. The extreme examples of this cause-effect relationship are exemplified by some published cases of (a) acute organization of AF into a MR AT gaps located in ablation lines which have been deployed just a few minutes before [75] and (b) gap-dependent MR ATs which can be induced shortly after apparently complete PV circumferential isolation [60]. The reason most frequently adduced to explain the presence of conduction gaps is immediate or delayed conduction recovery. Certainly, this is the only acceptable hypothesis when PV isolation and bidirectional conduction block across other additional lines was reliably confirmed [16-18, 62, 89] previously.

In turn, gap-related macroreentry is the most habitual type of MR PAFAT (65\%-96\%) [16, 21, 28, 58, 62, 89] and multiple gaps are habitually required $[36,71,73]$. In addition, a relevant proportion of focal or small-loop reentrant ATs have also been found spatially related to single (100\% of focal AT reported by Mesas et al. [16] and Luik et al. [60]) or double gaps (35\% of small-loop reentry reported by Deisenhofer et al. [15]). Pure PV tachycardias [73] after AF ablation as those published by Ouyang et al. [38] deserve a particular comment because they are a direct consequence of PV reconnections through gaps.

5.1.2. Identification of Gaps. Conduction gaps are viable tissue surrounded by permanent lesions; in consequence, they are located in incompletely ablated areas which can be revealed by magnetic resonance image techniques due to the presence of preserved atrial muscle bundles. It has been demonstrated that up to a maximum of $20 \%$ of the surface of the tissue where radiofrequency has been applied (areas usually marked by lesion dots on the 3D navigation systems) may correspond to viable tissue (not affected by gadolinium late-enhancement) [90]. To date, however, the efficacy of this approach to detect very small bundles of viable atrial myocytes has not been validated. An indirect way to suspect the presence of conduction gaps in ablation lines is the simultaneous achievement of AT interruption and bidirectional block across the line using a single or a few focal radiofrequency applications [38]. A detailed activation map can offer a more direct proof of the participation of gaps in a MR circuit when the activation front traverses perpendicularly the place where a prior ablation line was created $[21,60]$. The exact position of ablation lines relative to the AT circuit can be checked by side-by-side comparison of the AT activation map and the tridimensional reconstruction of the LA obtained during the initial AF ablation procedure, or with 
techniques of image integration implemented in modern 3D navigation systems, which allow for a more exact delineation of the spatial relationship between incomplete ablation lines and the circuit of the AT [91]. The most direct evidence which identifies a conduction gap is the presence of a single potential flanked by double potentials. The single potential (frequently fractionated) corresponds to the viable muscle and the double potentials to the ablation line lying at both sides of the gap [16].

5.1.3. Conduction Properties of Gaps. Noncontact mapping technology has provided greatly detailed images of the activation wavefront propagation across an ablation line through a gap: the activation front narrows upon reaching the gap and widens again at the other side of the line [92]. However, electrical conduction through discontinuous ablation lesions is a complex phenomenon influenced by the width of the gap, its geometry, and possibly the time elapsed since the lesions were created. In this sense, certain experimental models suggests that there is a minimum width necessary for gaps to conduct (habitually $3 \mathrm{~mm}$ [93]). Besides, the gap size can modulate the conduction velocity of the wavefront crossing it, in such a way that the narrower the gap, the slower the conduction velocity [93]. As a result, small gaps are usually characterized by long-duration and very fractionated potentials small gaps usually showing [9395]. The overall delay is not exceedingly great in comparison to normal tissue [94] and has been deemed to lack clinical relevance, insofar as small gaps are still capable of relatively fast conduction [38]. The solid results of Melby et al. [95] reaffirm both ideas: only very small gaps tend to manifest features of slow conduction (as a matter of fact, fragmented electrograms are a prominent distinctive mark of conduction gaps in the clinical setting $[16,38,58])$, and the overall conduction velocity impairment is small, allowing for effective conduction of very rapid paced rhythms and even AF.

On the other hand, conduction properties of gaps depend not only on their width but also on their geometrical configuration: angled or "L-" shaped gaps are much less likely to conduct than bifurcarted ("Y-" shaped) or straight gaps [65]. In general, sodium-channel blockers are effective in preventing gap-related electrical conduction in experimental models [65].

5.1.4. Frequent Locations of Gaps. Rostock et al. [87] reported the distribution of sites of conduction recovery in roof lines and mitral isthmus lines: $66 \%$ of gaps in the mitral isthmus line were localized in the upper portion, close to the left inferior pulmonary vein and the left atrial appendage; 54\% of gaps in the roof line were found in close relation to the right superior pulmonary vein ostium. Both preferential sites are the result of anatomical structures that condition a worse stability of the catheter and areas of greater wall thickness more difficult to ablate with transmural lesions. Similarly, Mesas et al. [16] found also a preferential location of gaps near the septum and the superior segment of the lateral left atrium, in both cases around the PV antra. Chang et al. [60] reported that the preferential location was another difficult place: the left atrial appendage ridge. However, Ouygang et al. [38] did not find any preferential distribution for 32 conduction gaps in circumferential lines around the PV ostia, in spite of the fact that gaps were approximately twice as frequent around the left PV.

\subsection{Critical Isthmus}

5.2.1. Anatomic Description. An isthmus can be defined as the narrowest part of the circuit. This definition involves two elements which need a precise characterization. Firstly, the course of the activation front defined as entirely as possible by the middle of activation mapping, entrainment mapping, noncontact mapping or analogous techniques; secondly, the presence of conduction barriers delimiting the anatomic channel through which the activation front traverses. These boundaries can be normal anatomic structures, scar areas, areas of conduction block (double potentials) [96], or previously ablated areas. Jaïs et al. [97] published an interesting study in which they identified critical isthmuses on the basis of an exclusively anatomic concept: an isthmus is configured by two lateral barriers and a corridor of normally excitable atrial tissue between them. These authors also demonstrated that a line of ablation transecting these isthmi is an adequate therapy for most macroreentrant AT. Obviously, this approach can be resorted to if the ATs is not stable enough as to resist a detailed entrainment mapping [98] and the participation of the isthmus in the circuit can be demonstrated at least with activation mapping. However, Ouyang et al. [99] showed with conclusive data that isthmi are usually narrow and the typical electrograms registered within them are of very low voltage amplitude (generally $<0.5 \mathrm{mV}$ ) and multicomponent or fragmented. As a result, isochronal and activation maps are specially limited to characterize them, because it is often really difficult to assign a reliable local activation time to such low-voltage and fragmented electrograms. Furthermore, purely anatomic isthmi are not always the feeblest part of the circuit. For example, the so-called mitral isthmus can be specially difficult to block bidirectionally [27], and this difficulty has led some authors to propose an alternative approach: the "anterior (or superior) line". This line, traced from the mitral annulus to either superior PV, blocks the entire anterior aspect of the left atrium [100, 101].

5.2.2. The Critical Isthmus from a Functional Point of View. De Ponti et al. [74] introduced a simple and elegant refinement of the conventional colour-coded activation map as a tool to help localize the zone of "diastolic" activation of the circuit. This strategy consists in a specific parameters setting of the $3 \mathrm{D}$ navigation system such that the location of the transition "purple-red" or "purple-white" (the traditional point where "head meets tail") limit identifies the position of the diastolic isthmus, which, in comparison to the systolic isthmus, is more frequently characterized by low-amplitude potentials and slow conduction [74] and corresponds almost always to the most vulnerable part of the circuit. 
Entrainment mapping, in turn, is the most consistent method to determine whether a given point in the atrium belongs to the circuit or not $[102,103]$. Isthmi can be defined with mere entrainment criteria [104] as those areas presenting concealed entrainment (defined as identical P-wave morphology and intra-atrial activation sequence during pacing and during AT), first postpacing interval equal to AT CL and a delay between the stimulus artifact and the elicited activation front of at least $40 \mathrm{msec}$. It seems clear, therefore, that activation mapping and entrainment mapping are complementary strategies [105] to localize the circuit and its course, boundaries, isthmi, slow-conduction areas, and, specially, sites where ablation has more chances to be effective, as Bogun et al. [104] studied in an excellent work only limited by the low number of cases included. Being it so, the true critical isthmus is not only a narrow corridor anatomically defined, but, above all, a site where the tachycardia is interrupted and rendered noninducible with the minimal number of radiofrequency applications. These sites hold some ancillary features which contribute to their identification: long activation times, split or fragmented electrograms, diastolic potentials and matching stimulus Pwave and electrogram P-wave intervals [104].

In the particular setting of PAFAT, isthmi can be constituted merely by gaps or by the interaction between ablated areas, preexisting scar areas and anatomic structures. Both an exclusive anatomical approach $[15,16,18,21,22]$, and a more functional $[17,37,61]$ characterization have been used to detect isthmi under those circumstances.

\subsection{Low-Voltage Areas and Slow-Conduction Zones}

5.3.1. Preexisting and Iatrogenic Scar Zones and Areas of Slow Conduction. Whereas patchy fibrosis and increased concentration of type I collagen have been observed in patients with lone paroxismal AF [106], significant scar areas (characterized by very low voltage, $\leq 0,1 \mathrm{mV}$ ) have not been described in this group of patients [107]. Some authors have postulated the origin of abnormal atrial zones (LVZs, slow-conduction and scar areas) to be either totally iatrogenic or the result of the interaction between incomplete radiofrequency lesions and specific anatomic structures (ligament of Marshall, autonomic ganglia) [58, 108]. However, there are important cumulative data supporting the existence of these abnormal areas independently of radiofrequency lesions. Taclas et al. [90] noticed that late gadolinium enhancement was sometimes detectable in locations where radiofrequency had not been applied. Moreno-Reviriego et al. [109] demonstrated the presence of a dense scar (characterized by absence of capture at maximal paced impulse output) or low-voltage area in 10 of 16 patients with persistent/long lasting FA. Verma et al. [110] detected scar areas in $6 \%$ of AF patients and demonstrated the role of these areas as independent predictors of AF recurrences. Lo et al. [41] investigated the progressive decrease in the mean LA voltage and increase in the extension of low-voltage zones (subtracted the contribution of ablated areas) in patients with AF recurrences after PVI. Lin et al. [111] evaluated the role of areas characterized by functional conduction block and low voltage in delimiting slow-conduction isthmi as a common mechanism for right atrial flutter and fibrillation. Cummings et al. [34] appreciated that preexisting scar areas may act as an additional substrate for PAFAT because PV reisolation alone was less efficacious to prevent recurrences in patients with scar areas than in those without them. Jais et al. [64] found that some flutter circuits were constituted by areas of slow conduction distant from PV ostia and not targeted by prior ablation. Yoshida et al. [62] concluded that radiofrequency lesions cannot be directly linked to at least $30 \%$ of AT that appear late after AF ablation because these tachycardias were not adjacent to ablation sites. In addition, as previously discussed, there is still a lack of demonstrative evidence that slow conduction could be attributed to RF lesions. For example, in spite of the complex conduction properties manifested by partially ablated areas, slow conduction was ruled out by Chorro et al. [112] as the mechanism of the prolonged conduction time measured near radiofrequency lesions because it could be always explained by the conduction detour of the wavefront around the lesion. Besides, it remains without explanation why RF is specially (or exclusively) prone to beget slow conduction and iatrogenic arrhythmias in the LA and not in other substrates such as ventricular scars or RA flutters [73].

5.3.2. Scars, Low-Voltage Zones, Slow-Conduction Areas, and Atrial Tachycardias. Independently of their origin, these abnormal areas may play a role in PAFAT for several reasons. First of all, LVZs are an integral part of the critical isthmus of most ATs. Secondly, LVZs and scars can simply make up the lateral boundaries delimiting a MR circuit, as is the case when a perimitral flutter is induced immediately after circumferential PVI [60] or after linear ablation at the roof, perhaps because the circuit is confined within these barriers and stabilized preventing short circuiting [21]. This constraining effect of natural and iatrogenic lateral barriers is most probably necessary for the maintenance of circuits unrelated to ablated zones [64]. There exist a more intricate cause-effect relation between areas of slow conduction and small-loop or localized reentrant AT, because when reentrant circuits are small in size, the phenomenon of slow electrical conduction acquires its most crucial relevance. Typically, small-loop reentrant circuits appear near previous ablation lesions and in places to which a special relevance for $\mathrm{AF}$ maintenance is commonly assigned, such as the PV antra $[16,21,37]$ or the LAA opening $[15,46,113]$. The critical component of these circuits is a narrow isthmus showing typical low-amplitude fractionated electrograms which span a great part of the CL, indicating slow conduction [37]. In the most extreme examples [15] fractionated potentials lasting up to $140 \mathrm{~ms}$ and occupying $60 \%$ of the TCL or even the entirety of the CL can be registered $[68,81]$. Deisenhoffer et al. [15] revealed something as important as frequently neglected: very slow-conduction areas are not located across ablation lines, on the contrary, they are simply adjacent to them. Consequently, it is reasonable to hypothesize that, if these slow-conduction areas existed 
before radiofrequency applications, they could have served as substrate for small and very rapidly rotating circuits implicated in the maintenance of AF. Posteriorly, RF lines could have modified the electrical properties of the circuit, for example, increasing its size and CL. The final result would be the creation of the mechanism of organized ATs as a consequence of the interaction between a previous abnormal substrate responsible for AF maintenance and radiofrequency lesions [73].

5.4. PV Reconnection and PV-Related Triggering Foci. PVrelated foci of ectopic activity implicated in AF initiation and maintenance seem to play a relevant role in triggering organized PAFAT, although the participation of extra PV triggers should not be underestimated.

As noted before, PVI is the only element of all the AF ablation techniques clearly associated with a reduced incidence of PAFAT. After AF ablation, the persistence of PVI is also fundamental for sinus rhythm maintenance. The most relevant piece of evidence was provided by the great proportion of patients in whom PV reconnection was demonstrated during the PAFAT study and ablation. Ouyang et al. [38] and Chun et al. [114] addressed the problem of PV reconnection in both case series of PAFAT after catheter-guided PVI and surgical Maze, respectively. Their results were incontrovertible: $80 \%$ of patients with AT recurrences after catheter ablation and $88 \%$ of patients with AT after Maze procedure presented PV reconnection. Similarly, according to a recent study by Sy et al. [115], in the group of patients requiring a second ablation procedure after PVI (48.3\% presented recurrences in form of organized PAFAT), $82 \%$ of the PV were reconnected. Other authors $[33,34,36,39]$ have also indirectly proved the cause-effect relation between the presence of venoatrial reconnections and PAFAT. In addition, the contribution of ectopic activity arising from the PV to the initiation of organized AT is also proved by the capital importance of PV reisolation to reduce AT recurrences. For example, Cummings et al. [34] evaluated the effect of PV reisolation alone to treat LA flutters following a previous PVI procedure and obtained interesting results: $61 \%$ of patients remained free of arrhythmia recurrences off antiarrhythmics and an additional $21 \%$ on antiarrhythmics. The importance of abolishing the contribution of PV-related triggers was reported by Wazni et al. [116] as well. Patients with coexistent $\mathrm{AF}$ and typical right atrial flutter were included in this study and underwent PVI isolation without concomitant bidirectional cavotricuspid isthmus blockade, which was curative in most of them. Therefore, in the light of all these data PV reconnection remains the milestone of the pathologic process which ultimately leads to PAFAT occurrence, to such an extent that a certain dependence of organized AT on PV triggering foci has to be assumed. The question is whether this relationship is direct or indirect, or in other words, whether PV ectopics are able to initiate organized AT with or without an intermediate period of AF $[116,117]$. Both possibilities are likely but it must be noted that, in a simulation study, Gong et al. [118] demonstrated that the atria are more vulnerable to premature beats arising from the PVs than from other locations and that the vulnerable window for atrial flutter/AF induction (the range of different pacing CL and extrastimuli coupling intervals) is markedly smaller for right atrial foci in comparison to $\mathrm{PV}$ foci.

Two reasonable consequences can be drawn from the close relationship between PV reconnection and PAFAT. Firstly, PV reisolation should be considered the first procedural step when a PAFAT ablation is undertaken [14]. Secondly, all measures aiming at reducing the risk of PV reconnection have to be implemented. For example, it is commonly reckoned today that a mere anatomic atrial ablation guided by electroanatomic mapping systems is unreliable in achieving a complete PVI [24, 119]. Consequently, PVI should be assessed routinely by a circularmapping-guided catheter approach, taking into account that critical areas such as the carina between ipsilateral veins have to be targeted for ablation almost always [120]. Finally, many laboratories have adopted the use of general anesthesia for AF ablation procedures in view of the lower probability of PV reconnection reported by Di Biase et al. [121] or have started to employ magnetic robotic navigation $[122,123]$. This new technology has been suggested to improve the catheter stability or to simplify the PVI technique.

\section{Results of Ablation}

In the previous paragraphs we have enumerated the great number of strategies which have been published to localize and characterize the mechanism of PAFAT. All of them should be considered complementary approaches, and their use should be conditioned to the operator experience and to the particular requirements and peculiarities of each AT in an individualized manner. Basically, our experience and the published results of other authors invite to consider that activation mapping should always be complemented with entrainment maneouvres and that the tridimensional image support afforded by electroanatomic navigation systems is in general advisable to optimize the results of ablation. The algorithm proposed by Knecht et al. [14] and developed by Jaïs et al. [61] deserves the utmost attention because it provides accurate rules to elucidate the most frequent PAFAT mechanisms laying emphasis on simple and precise criteria: PVI reisolation is performed first and then focal, macro-reentrant, and small-loop reentrant mechanisms are systematically sought after in this order.

In spite of the heterogeneity of techniques habitually resorted and the differences in the degree of mechanistic characterization of these arrhythmias, several authors have published convincing results (see Table 1) showing the success rate of PAFAT invasive treatment. The clinical PAFAT can be ablated almost always (70-100\% of individual AT). Patel et al. [72] and Deisenhoffer et al. [15] reported a somewhat lower percentage of success (61\% and 38\%, resp.) which may be explained because most of their patients presented several different ATs. However, recurrences of new organized ATs are not rare (up to $21 \%-44 \%$ ). Longterm sinus rhythm maintenance has not been conveniently 


\begin{tabular}{|c|c|c|c|c|c|c|c|c|c|c|c|c|c|}
\hline 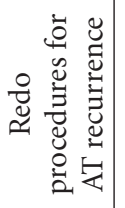 & \&̊ & 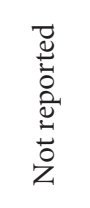 & 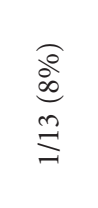 & \&̊ & 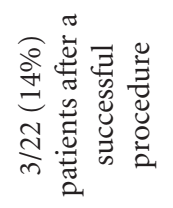 & 8̊ & 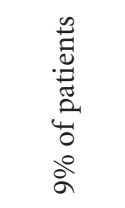 & 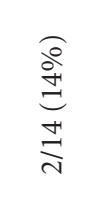 & $\stackrel{8}{\circ}$ & 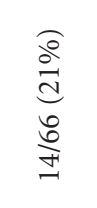 & 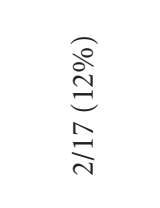 & 80 & Z̃ \\
\hline 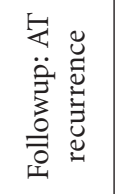 & 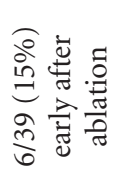 & $\begin{array}{l}\stackrel{\circ}{\circ} \\
\stackrel{\circ}{\circ} \\
\stackrel{\circ}{=}\end{array}$ & 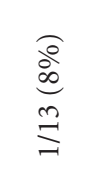 & $8^{\circ}$ & 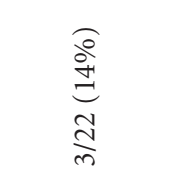 & \&े & 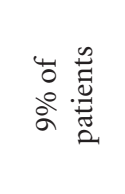 & $\frac{\substack{o \\
\frac{d}{d}}}{\stackrel{+}{d}}$ & $8^{\circ}$ & 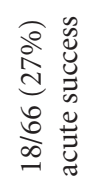 & 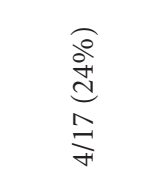 & 8̊ & $\frac{\widehat{o}}{\stackrel{\Xi}{\Xi}}$ \\
\hline 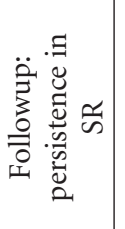 & 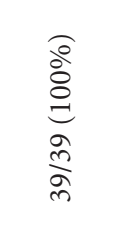 & 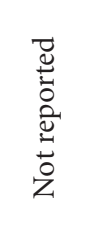 & $\underset{\substack{0 \\
\stackrel{0}{0}}}{\stackrel{m}{\Xi}}$ & $\begin{array}{l}\text { ò } \\
\stackrel{2}{0} \\
\frac{1}{a} \\
\frac{1}{a}\end{array}$ & 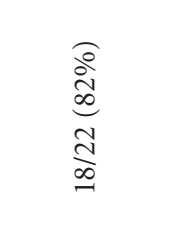 & 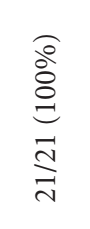 & 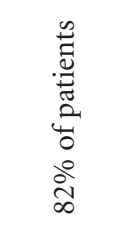 & 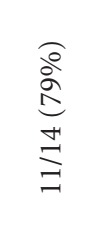 & 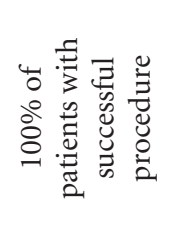 & $\begin{array}{l}\frac{\partial}{2} \\
\stackrel{1}{\infty} \\
\infty \\
\frac{\infty}{8} \\
0\end{array}$ & 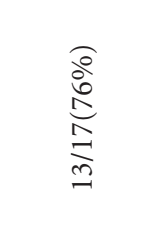 & $\begin{array}{l}\frac{0}{\infty} \\
\infty \\
\frac{\infty}{n} \\
\infty\end{array}$ & $\frac{\widehat{o}}{\frac{\partial}{a}}$ \\
\hline 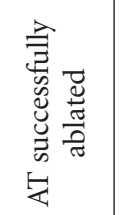 & 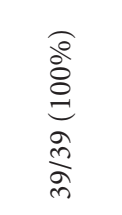 & 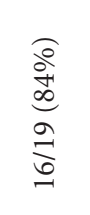 & 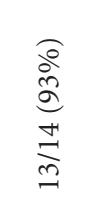 & $\begin{array}{l}\frac{0}{8} \\
\stackrel{\circ}{0} \\
\frac{1}{a}\end{array}$ & 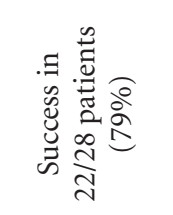 & 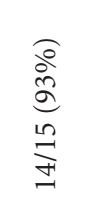 & 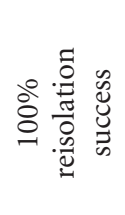 & 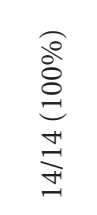 & $\begin{array}{l}\frac{a}{a} \\
\frac{1}{0} \\
\frac{1}{0} \\
0\end{array}$ & 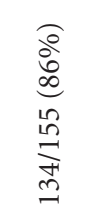 & 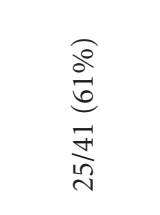 & $\underset{\substack{\infty \\
\stackrel{\infty}{\infty}}}{\stackrel{\infty}{\infty}}$ & 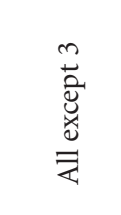 \\
\hline 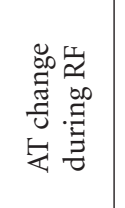 & 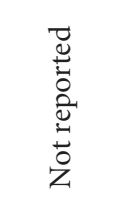 & 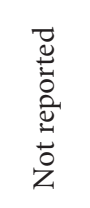 & 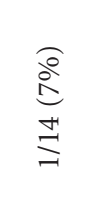 & $\stackrel{\circ}{\circ}$ & 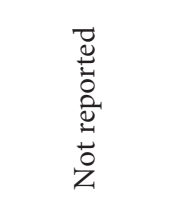 & 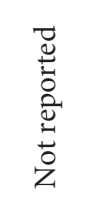 & 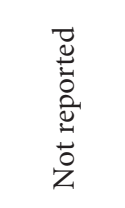 & 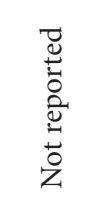 & 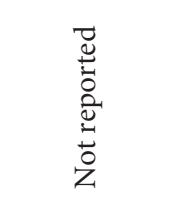 & 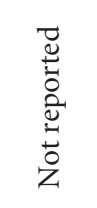 & 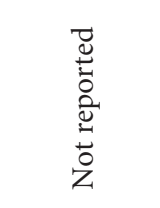 & 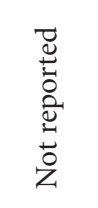 & 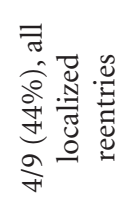 \\
\hline 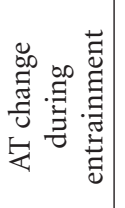 & 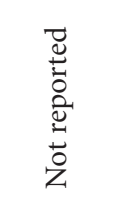 & 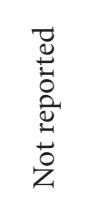 & 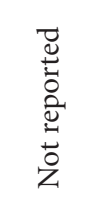 & 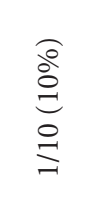 & 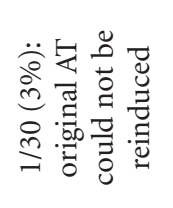 & 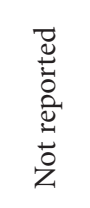 & 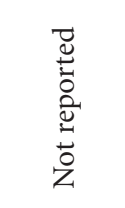 & 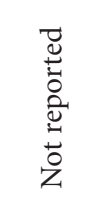 & 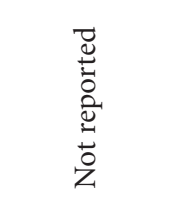 & 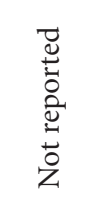 & 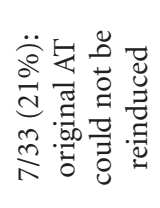 & 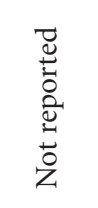 & 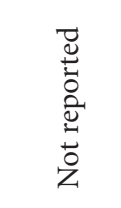 \\
\hline 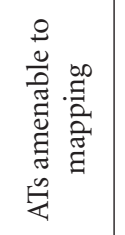 & 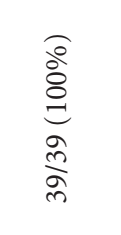 & 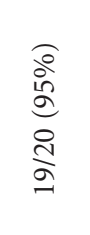 & 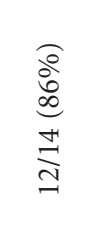 & 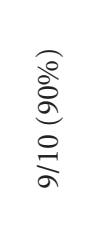 & 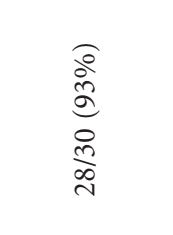 & $\begin{array}{l}\widehat{0} \\
\infty \\
\infty \\
0 \\
\stackrel{0}{n}\end{array}$ & 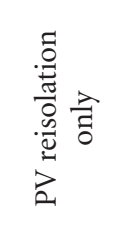 & 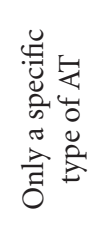 & $\begin{array}{l}\frac{a}{b} \\
\stackrel{0}{E} \\
\stackrel{0}{a}\end{array}$ & 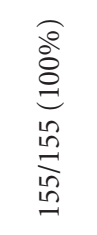 & 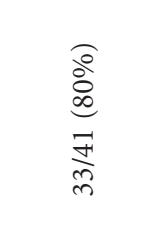 & 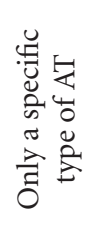 & 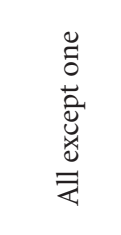 \\
\hline 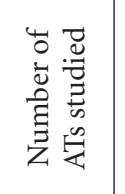 & సे & ㄱ. & $\exists$ & $\stackrel{-}{\circ}$ & 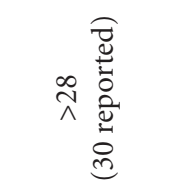 & $\triangleq$ & 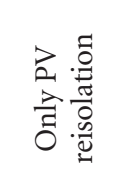 & $\Xi$ & $\triangleq$ & $\stackrel{\Omega}{\Omega}$ & $F$ & $\infty$ & 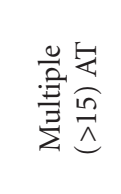 \\
\hline 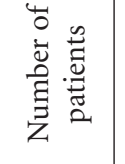 & (े) & $\stackrel{\circ}{\circ}$ & $\mathscr{m}$ & 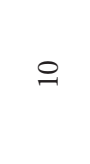 & $\stackrel{\infty}{\sim}$ & $\vec{\sim}$ & $\ddot{\sim}$ & $\Xi$ & $a$ & $\stackrel{\infty}{\triangleright}$ & $\triangleq$ & $\infty$ & $a$ \\
\hline 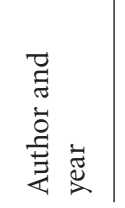 & 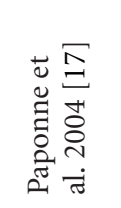 & 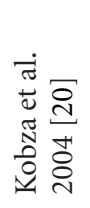 & 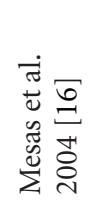 & 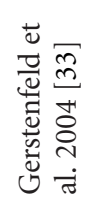 & 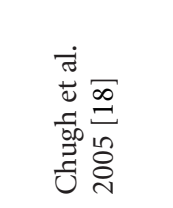 & 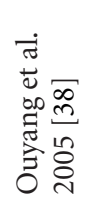 & 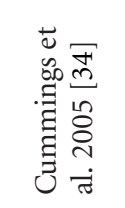 & 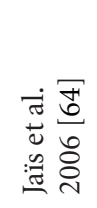 & 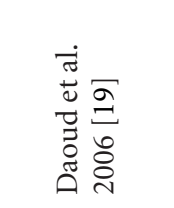 & 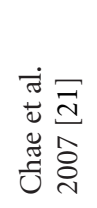 & 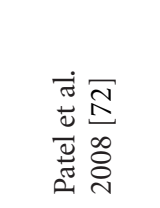 & 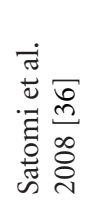 & 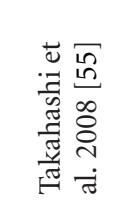 \\
\hline
\end{tabular}




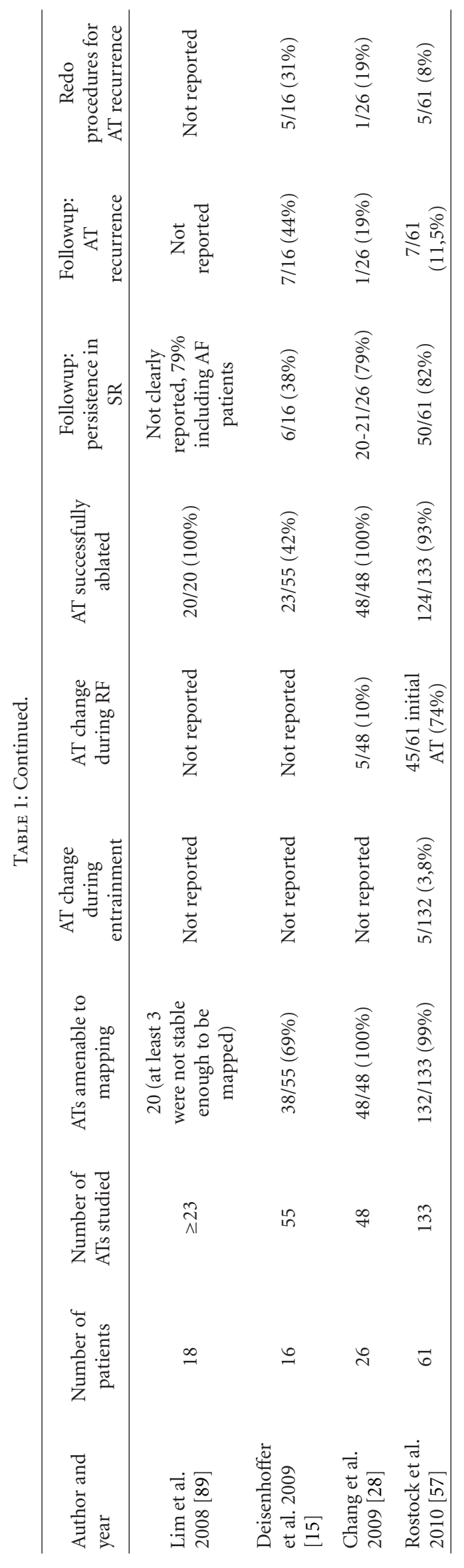


explored by the studies summarized in Table 1 because the time of fol-lowup after the PAFAT treatment was not long enough in most cases.

\section{Organized AT during AF Ablation}

AF termination is considered by some authors a desiderate but not always attainable objective of AF ablation. The distillation [14] of AF into organized AT has been judged a reasonable way to modify the substrate responsible for AF maintenance. However, the extensive ablation this objective usually demands, together with the controversial results which have apparently disproved its efficacy in terms of effective prevention of AF recurrences [124], has precluded a more widespread acceptation of this opinion. At all events, organization of AF into AT during ablation is an interesting phenomenon, worthy of mention because it has given rise to new hypothesis about AF maintenance mechanisms. Some of these organized ATs are not spontaneous. For example, Chang et al. [28] induced organized AT in $16.3 \%$ of patients immediately after circumferential PVI, most of them (76\%) were gap-related AT and macro-reentrant circuits around the mitral annulus or ipsilateral PVs were the most frequent. In fact, this inducibility guided strategy may be helpful to uncover gaps and latent AT circuits which could eventually acquire clinical relevance afterwards [18]. On the other hand, spontaneous ATs have much more interest from a mechanistic point of view because they could indicate the location of areas relevant for AF maintenance. Scharf et al. studied six cases of spontaneous conversion of AF into focal AT during left atrial ablation. All these ATs had a focal origin adjacent to ablated areas but unrelated to conduction gaps, and what is even more important, three of them had also an exact spatial correspondence with areas that seemed to anchor the fastest frequencies during $\mathrm{AF}$ (characterized by a CL during AF $30-40 \mathrm{~ms}$ shorter than the mean CL in adjacent zones). These focal sources of organized AT were correctly interpreted as slowed versions of very rapidly firing zones, the mechanism which had been probably modified by the ablation procedure. Only one of them manifested the typical behavior of an automatic focus but the mechanism was not investigated in great detail in the rest. A further and not less exciting insight into the hypothesis that $\mathrm{AF}$ and organized $\mathrm{AT}$ could be two sides of the same coin was provided by the study of Yoshida et al. [62] In this study the AF ablation strategy consisted in antral PVI and targeting of complex fractionated electrograms until AF converted to sinus rhythm or organized AT. There was a spectral component in the AF periodogram that matched the frequency of the resulting AT in $52 \%$ of patients who presented acute transformation of AF into AT. This proportion was higher (79\%) when the AF preriodogram was evaluated just before conversion to AT. This correspondence between the frequency of the resulting $\mathrm{AT}$ and one of the components of the AF periodogram suggests that the AT circuit is already present during AF in some way or another, but hidden behind the fibrillatory proccess and surpassed by the higher-frequency components of the AF spectrum of frequencies. As for the mechanism of these resulting ATs, 94\% were macro- or micro-reentry circuits, $70 \%$ of them located distant from the ablated areas. How crucial is the contribution of these lower-frequency drivers to the global fibrillatory phenomenon? It is evident that the lower-frequency components alone cannot result in fibrillatory activity once the higher-frequency components have been abolished. Nevertheless, it is not known whether the fibrillatory process requires not only the high-frequency drivers to be manifest but also the lower-frequency reentrant circuits to remain stable or what would happen if these subordinate components could be localized and ablated independently in the first instance.

Organization of AF into AT during extensive CAFEs ablation occurs in $36 \%$ of cases of paroxysmal AF and in $50 \%$ of persistent AF [125]. Different mechanisms of AT in this context were described in pioneer works [46] and have been recently revisited by Nam et al. [53], who have reported the following results: $30 \%$ perimitral MR, 30\% cavotricuspid isthmus-dependent flutter, $18 \%$ roof-dependent MR, $18 \%$ focal and $6 \%$ of unknown mechanism. These circuits are well-known, amenable to mapping, and commonly ablated in daily clinical practice, from whence comes the opinion that AF organization might be as good an outcome as termination [61].

\section{Conclusions}

The incidence of PAFAT is clinically relevant but depends on the ablation technique initially used to treat $\mathrm{AF}$ and on the existence of appropriate anatomic substrates, which sometimes exist before the ablation procedure. Both the mechanisms and elements constitutive of PAFAT circuits are well-known and have been repeatedly and consistently described. This fact, along with the high probability of success when these AT are targeted for ablation, and the low efficacy of antiarrhytmic drugs, implies that an invasive approach should be attempted if these AT became incessant, bad tolerated or do not disappear after a prudential observation period. However, it must be noted that the frequent coexistence of multiple mechanisms and several different types of PAFAT in the same patient determine the special complexity of these procedures, in which PV reisolation is the pivotal element when reconnection has occurred.

\section{References}

[1] H. Oral, C. Scharf, A. Chugh et al., "Catheter ablation for paroxysmal atrial fibrillation: segmental pulmonary vein ostial ablation versus left atrial ablation," Circulation, vol. 108, no. 19, pp. 2355-2360, 2003.

[2] H. Häissaguerre, P. Jais, M. Hocini et al., "Macro-reentrant atrial flutter following ablation of pulmonary veins, tricuspid and mitral isthmuses," Pacing and Clinical Electrophysiology, vol. 26, p. 970, 2003.

[3] H. Oral, B. P. Knight, and F. Morady, "Left atrial flutter after segmental ostial radiofrequency catheter ablation for pulmonary vein isolation," Pacing and Clinical Electrophysiology, vol. 26, no. 6, pp. 1417-1419, 2003. 
[4] L. Kanagaratnam, G. Tomassoni, R. Schweikert et al., "Empirical pulmonary vein isolation in patients with chronic atrial fibrillation using a three-dimensional nonfluoroscopic mapping system: long-term follow-up," Pacing and Clinical Electrophysiology, vol. 24, no. 12, pp. 1774-1779, 2001.

[5] J. Villacastín, N/ Pérez-Castellano, J Moreno et al., "Left atrial flutter after radiofrequency cathter ablation of focal atrial fibrillation," Journal of Cardiovascular Electrophysiology, vol. 14, no. 4, pp. 417-421, 2003.

[6] N. Saoudi, F. Cosío, A. Waldo et al., "A classification of atrial flutter and regular atrial tachycardia according to electrophysiological mechanisms and anatomical bases: a statement from a joint expert group from the working group of arrhythmias of the European society of cardiology and the North American society of pacing and electrophysiology," European Heart Journal, vol. 22, no. 14, pp. 1162-1182, 2001.

[7] F. Anselme, "Macroreentrant atrial tachycardia: pathophysiological concepts," Heart Rhythm, vol. 5, no. 6, pp. S18-S21, 2008.

[8] J. P. Daubert, "Iatrogenic left atrial tachycardias: where are we?" Journal of the American College of Cardiology, vol. 50, no. 18, pp. 1788-1790, 2007.

[9] E. P. Gerstenfeld and F. E. Marchlinski, "Mapping and ablation of left atrial tachycardias occurring after atrial fibrillation ablation," Heart Rhythm, vol. 4, no. 3, pp. S65S72, 2007.

[10] K. Satomi, "Electrophysiological characteristics of atrial tachycardia after pulmonary vein isolation of atrial fibrillation," Circulation Journal, vol. 74, no. 6, pp. 1051-1058, 2010.

[11] J. V. Wylie and M. E. Josephson, "Atrial tachyarrhythmias after atrial fibrillation ablation procedures: trading one tachycardia for another?" Journal of Cardiovascular Electrophysiology, vol. 17, no. 4, pp. 374-376, 2006.

[12] F. Morady, H. Oral, and A. Chugh, "Diagnosis and ablation of atypical atrial tachycardia and flutter complicating atrial fibrillation ablation," Heart Rhythm, vol. 6, no. 8, pp. S29S32, 2009.

[13] G. D. Veenhuyzen, S. Knecht, M. D. O’Neill et al., "Atrial tachycardias encountered during and after catheter ablation for atrial fibrillation: part I: classification, incidence, management," Pacing and Clinical Electrophysiology, vol. 32, no. 3, pp. 393-398, 2009.

[14] S. Knecht, G. Veenhuyzen, M. O’Neill, M. Wright, I. Nault et al., "Atrial tachycardias encountered during and after catheter ablation for atrial fibrillation. Part II: mapping and ablation," Pacing and Clinical Electrophysiology, vol. 32, no. 3, pp. 528538, 2009.

[15] I. Deisenhofer, H. Estner, B. Zrenner et al., "Left atrial tachycardia after circumferential pulmonary vein ablation for atrial fibrillation: incidence, electrophysiological characteristics, and results of radiofrequency ablation," Europace, vol. 8, no. 8, pp. 573-582, 2006.

[16] C. E. Mesas, C. Pappone, C. C. E. Lang et al., "Left atrial tachycardia after circumferential pulmonary vein ablation for atrial fibrillation," Journal of the American College of Cardiology, vol. 44, no. 5, pp. 1071-1079, 2004.

[17] C. Pappone, F. Manguso, G. Vicedomini et al., "Prevention of iatrogenic atrial tachycardia after ablation of atrial fibrillation," Circulation, vol. 110, no. 19, pp. 3036-3042, 2004.

[18] A. Chugh, H. Oral, K. Lemola et al., "Prevalence, mechanism, and clinical significance of macrorreentrant atrial tachycardia during and following left atrial ablation for atrial fibrillation," Heart Rhythm, vol. 2, no. 5, pp. 464-471, 2005.
[19] E. G. Daoud, R. Weiss, R. Augostini et al., "Proarrhythmia of circumferential left atrial lesions for management of atrial fibrillation," Journal of Cardiovascular Electrophysiology, vol. 17, pp. 157-165, 2006.

[20] R. Kobza, G. Hindricks, H. Tanner et al., "Late recurrent arrhythmias after ablation of atrial fibrillation: incidence, mechanisms, and treatment," Heart Rhythm, vol. 1, no. 6, pp. 676-683, 2004.

[21] S. Chae, H. Oral, E. Good et al., "Atrial tachycardia after circumferential pulmonary vein ablation of atrial fibrillation. Mechanistic insights, results of catheter ablation and risk factors for recurrence," Journal of the American College of Cardiology, vol. 50, no. 18, pp. 1781-1787, 2007.

[22] A. Chugh, R. Latchamsetty, H. Oral et al., "Characteristics of cavotricuspid isthmus-dependent atrial flutter after left atrial ablation of atrial fibrillation," Circulation, vol. 113, no. 5, pp. 609-615, 2006.

[23] F. Kilicaslan, A. Verma, H. Yamaji et al., "The need for atrial flutter ablation following pulmonary vein antrum isolation in patients with and without previous cardiac surgery," Journal of the American College of Cardiology, vol. 45, no. 5, pp. 690-696, 2005.

[24] M. R. Karch, B. Zrenner, I. Deisenhofer et al., "Freedom from atrial tachycarrhythmias after catheter ablation of atrial fibrillation. A randomized comparison between 2 current ablation strategies," Circulation, vol. 111, no. 22, pp. 28752880, 2005.

[25] C. Pappone, G. Oreto, S. Rosanio et al., "Atrial electroanatomic remodeling after circumferential radiofrequency vein ablation: efficacy of an anatomic approach in a large cohort of patients with atrial fibrillation," Circulation, vol. 104, no. 21, pp. 2539-2544, 2001.

[26] J.-I. Choi, H.-N. Pak, J. S. Park et al., "Clinical significance of early recurrences of atrial tachycardia after atrial fibrillation ablation," Journal of Cardiovascular Electrophysiology, vol. 21, no. 12, pp. 1331-1337, 2010.

[27] R. Anousheh, N. S. Sawhney, M. Panutich, C. Tate, W. C. Chen, and G. K. Feld, "Effect of mitral isthmus block on development of atrial tachycardia following ablation for atrial fibrillation," Pacing and Clinical Electrophysiology, vol. 33, no. 4, pp. 460-468, 2010.

[28] S.-L. Chang, Y.-J. Lin, C.-T. Tai et al., "Induced atrial tachycardia after circumferential pulmonary vein isolation of paroxysmal atrial fibrillation: electrophysiological characteristics and impact of catheter ablation on the follow-up results," Journal of Cardiovascular Electrophysiology, vol. 20, no. 4, pp. 388-394, 2009.

[29] A. G. Brooks, M. K. Stiles, J. Laborderie et al., "Outcomes of long-standing persistent atrial fibrillation ablation: a systematic review," Heart Rhythm, vol. 7, no. 6, pp. 835-846, 2010.

[30] H. Oral, B. P. Knight, H. Tada et al., "Pulmonary vein isolation for paroxysmal and persistent atrial fibrillation," Circulation, vol. 105, no. 9, pp. 1077-1081, 2002.

[31] N. F. Marrouche, T. Dresing, C. Cole et al., "Circular mapping and ablation of the pulmonary vein for treatment of atrial fibrillation: impact of different catheter technologies," Journal of the American College of Cardiology, vol. 40, no. 3, pp. 464-474, 2002.

[32] A. Verma, N. F. Marrouche, and A. Natale, "Pulmonary vein antrum isolation: intracardiac echocardiography-guided technique," Journal of Cardiovascular Electrophysiology, vol. 15, no. 11, pp. 1335-1340, 2004. 
[33] E. P. Gerstenfeld, D. J. Callans, S. Dixit et al., "Mechanism of organized left atrial tachycardias occurring after pulmonary vein ablation," Circulation, vol. 110, no. 11, pp. 1351-1357, 2004.

[34] J. Cummings, R. Schweikert, W. Saliba et al., "Left atrial flutter following pulmonary vein antrum isolation with radiofrequency energy: linear lesions or repeat isolation," Journal of Cardiovascular Electrophysiology, vol. 16, no. 3, pp. 293-297, 2005.

[35] S. Themistoclakis, R. A. Schweikert, W. I. Saliba et al., "Clinical predictors and relationship between early and late atrial tachyarrhythmias after pulmonary vein antrum isolation," Heart Rhythm, vol. 5, no. 5, pp. 679-685, 2008.

[36] K. Satomi, D. Bänsch, R. Tilz et al., "Left atrial and pulmonary vein macrorreentrant tachycardia associated with double conduction gaps: a novel type of man-made trachycardia after circumferential pulmonary vein isolation," Heart Rhythm, vol. 5, no. 1, pp. 43-51, 2008.

[37] D. Shah, H. Sunthorn, H. Burri et al., "Narrow, slowconducting isthmus dependent left atrial reentry developing after ablation for atrial fibrillation: ECG characterization and elimination by focal RF ablation," Journal of Cardiovascular Electrophysiology, vol. 17, no. 5, pp. 508-515, 2006.

[38] F. Ouyang, M. Antz, S. Ernst et al., "Recovered pulmonary vein conduction as a dominant factor for recurrent atrial tachycarrhythmias after complete circular isolation of the pulmonary veins. Lesson from double Lasso technique," Circulation, vol. 111, pp. 127-135, 2005.

[39] E. P. Gerstenfeld, D. J. Callans, W. Sauer, J. Jacobson, and F. E. Marchlinski, "Reentrant and nonreentrant focal left atrial tachycardias occur after pulmonary vein isolation," Heart Rhythm, vol. 2, no. 11, pp. 1195-1202, 2005.

[40] M. K. Stiles, B. John, C. X. Wong et al., "Paroxysmal lone atrial fibrillation is associated with an abnormal atrial substrate. Characterizing the 'second factor," Journal of the American College of Cardiology, vol. 53, no. 14, pp. 11821191, 2009.

[41] L.-W. Lo, C.-T. Tai, Y.-J. Lin et al., "Progressive remodeling of the atrial substrate- a novel finding from consecutive voltage mapping in patients with recurrence of atrial fibrillation after catheter ablation," Journal of Cardiovascular Electrophysiology, vol. 18, no. 3, pp. 258-265, 2007.

[42] M. Porter, W. Spear, J. G. Akar et al., "Prospective study of atrial fibrillation termination during ablation guided by auutomated detection of fractionated electrograms," Journal of Cardiovascular Electrophysiology, vol. 19, pp. 613-620, 2008.

[43] N. Sahwney, R. Anousheh, W. Chen, and G. K. Feld, "Circumferential pulmonary vein ablation with additional linear ablation results in an increased incidence of left atrial flutter compared with segmental pulmonary vein isolation as an initial approach to ablation of paroxismal atrial fibrillation," Circulation: Arrhythmia and Electrophysiology, vol. 3, pp. 243-248, 2010.

[44] F. Gaita, D. Caponi, M. Scaglione et al., "Long-term clinical results of 2 different ablation strategies in patients with paroxismal and persistent atrial fibrillation," Circulation: Arrhythmia and Electrophysiology, vol. 1, pp. 269-275, 2008.

[45] K. Nademanee, J. McKenzie, E. Kosar et al., "A new approach for catheter ablation of atrial fibrillation: mapping of the electrophysiologic substrate," Journal of the American College of Cardiology, vol. 43, no. 11, pp. 2044-2053, 2004.

[46] M. Haïssaguerre, P. Sanders, M. Hocini et al., "Catheter ablation of long-lasting persistent atrial fibrillation: critical structures for termination," Journal of Cardiovascular Electrophysiology, vol. 16, no. 11, pp. 1125-1137, 2005.

[47] H. Oral, A. Chugh, E. Good et al., "Radiofrequency catheter ablation of chronic atrial fibrillation guided by complex electrograms," Circulation, vol. 115, no. 20, pp. 2606-2612, 2007.

[48] H. Oral, A. Chugh, K. Yoshida et al., "A randomized assessment of the incremental role of ablation of complex fractionanted atrial electrograms after pulmonary vein isolation for long-lasting persistent atrial fibrillation," Journal of the American College of Cardiology, vol. 53, no. 9, pp. 782789, 2009.

[49] H. Oral, A. Chugh, E. Good et al., "Randomized comparison of encircling and nonencircling left atrial ablation for chronic atrial fibrillation," Heart Rhythm, vol. 2, no. 11, pp. 11651172, 2005.

[50] H. L. Estner, G. Hessling, G. Ndrepepa et al., "Acute effects and long-term outcome of pulmonary vein isolation in combination with electrogram-guided substrate ablation for persistent atrial fibrillation," American Journal of Cardiology, vol. 101, no. 3, pp. 332-337, 2008.

[51] C. S. Elayi, A. Verma, L. Di Biase et al., "Ablation for longstanding atrial fibrillation: results from a randomized study comparing three different strategies," Heart Rhythm, vol. 5, no. 12, pp. 1568-1664, 2008.

[52] K. Nademanee, M. C. Schwab, E. M. Kosar et al., "Clinical outcomes of catheter substrate ablation for high-risk patients with atrial fibrillation," Journal of the American College of Cardiology, vol. 51, no. 8, pp. 843-849, 2008.

[53] G.-B. Nam, E.-S. Jin, H.-O Choi et al., "Mechanism of regular atrial tachyarrhythmias during combined pulmonary vein isolation and complex fractionated electrogram ablation in patients with atrial fibrillation," Circulation Journal, vol. 74, no. 3, pp. 434-441, 2010.

[54] T. Rostock, D. Steven, B. Hoffmann et al., "Chronic atrial fibrillation is a biatrial arrhythmia: data from catheter ablation of chronic atrial fibrillation aiming arrhythmia termination using a sequential ablation approach," Circulation: Arrhythmia and Electrophysiology, vol. 1, no. 5, pp. 344-353, 2008.

[55] Y. Takahashi, M. D. O’Neill, M. Hocini et al., "Characterization of electrograms assotiated with termination of chronic atrial fibrillation by catheter ablation," Journal of the American College of Cardiology, vol. 51, no. 10, pp. 10031010, 2008.

[56] M. Haïssaguerre, P. Sanders, M. Hocini et al., "Catheter ablation of long-lasting persistent atrial fibrillation: clinical outcome and mechanisms of susequent arrythmias," Journal of Cardiovascular Electrophysiology, vol. 16, pp. 1138-1147, 2005.

[57] T. Rostock, I. Drewitz, D. Steven et al., "Characterization, mapping, and catheter ablation of recurrent atrial tachycardias after stepwise ablation of long-lasting persistent atrial fibrillation," Circulation: Arrhythmia and Electrophysiology, vol. 3, no. 2, pp. 160-169, 2010.

[58] L. Zheng, Y. Yao, S. Zhang et al., "Organized left atrial tachycarrhythmia during stepwise linear ablation for atrial fibrillation," Journal of Cardiovascular Electrophysiology, vol. 20, pp. 499-506, 2009.

[59] Y. Takahashi, A. Takahashi, S. Miyazaki et al., "Electrophysiological characteristics of localized reentrant atrial tachycardia occurring after cathter ablation of long-lasting persistent atrial fibrillation," Journal of Cardiovascular Electrophysiology, vol. 20, pp. 623-926, 2009. 
[60] A. Luik, M. Merkel, T. Riexinger, R. Wondraschek, and C. Schmitt, "Persistent atrial fibrillation converts to common type atrial flutter during CFAE ablation," Pacing and Clinical Electrophysiology, vol. 33, no. 3, pp. 304-308, 2010.

[61] P. Jaîs, S. Matsuo, S. Knecht et al., "A deductive mapping strategy for atrial tachycardia following atrial fibrillation ablation: importance of localized reentry," Journal of Cardiovascular Electrophysiology, vol. 20, no. 5, pp. 480-491, 2009.

[62] K. Yoshida, A. Chugh, M. Ulfarsson et al., "Relationship between the spectral characteristics of atrial fibrillation and atrial tachycardias that occur after catheter ablation of atrial fibrillation," Heart Rhythm, vol. 6, no. 1, pp. 11-17, 2009.

[63] E. Bertaglia, G. Stabile, G. Senatore et al., "Predictive value of early atrial tachyarrhythmias recurrence after circumferential anatomical pulmonary vein ablation," Pacing and Clinical Electrophysiology, vol. 28, no. 5, pp. 366-371, 2005.

[64] P. Jaïs, P. Sanders, L. F. Hsu et al., "Flutter localized to the anterior left atrium after catheter ablation of atrial fibrillation," Journal of Cardiovascular Electrophysiology, vol. 17, no. 3, pp. 279-285, 2006.

[65] F. J. Perez, M. A. Wood, and C. M. Schubert, "Effects of gap geometry on conduction through discontinuous radiofrequency lesions," Circulation, vol. 113, no. 14, pp. 17231729, 2006.

[66] M. Haïssaguerre, M. Hocini, P. Sanders et al., "Localized sources maintaining atrial fibrillation organized by prior ablation," Circulation, vol. 113, no. 5, pp. 616-625, 2006.

[67] S. Higa, C.-T. Tai, Y.-J. Lin et al., "Focal atrial tachycardia. New insight from noncontact mapping and catheter ablation," Circulation, vol. 109, no. 1, pp. 84-91, 2004.

[68] P. Sanders, M. Hocini, P. Jaïs et al., "Characterization of focal atrial tachycardia using high-density mapping," Journal of the American College of Cardiology, vol. 46, no. 11, pp. 20882099, 2005.

[69] R. R. Tilz, K. R. J. Chun, B. Schmidt et al., "Catheter ablation of long-lasting persistent atrial fibrillation: a lesson from circumferential pulmonary vein isolation," Journal of Cardiovascular Electrophysiology, vol. 21, pp. 1085-1093, 2010.

[70] T. A. Irtel and E. Delacrétaz, "Intra-atrial reentrant tachycardia with ambiguous data from activation mapping: what to do next?" Heart Rhythm, vol. 2, no. 7, pp. 780-781, 2005.

[71] G. D. Veenhuyzen and F. R. Quinn, "Mind the gap! An atrial tachycardia after catheter ablation for atrial fibrillation," Journal of Cardiovascular Electrophysiology, vol. 20, no. 8, pp. 949-951, 2009.

[72] A. M. Patel, A. D’Avila, P. Neuzil et al., "Atrial tachycardia after ablation of persistent atrial fibrillation. Identification of the critical isthmus with a combination of multielectrode activation mapiing and targeted entrainment mapping," Circulation: Arrhythmia and Electrophysiology, vol. 1, no. 1, pp. 14-22, 2008.

[73] J. L. Merino, "Slow conduction and flutter following atrial fibrillation ablation: proarrhythmia or unmasking effect of radiofrequency application?" Journal of Cardiovascular Electrophysiology, vol. 17, no. 5, pp. 516-519, 2006.

[74] R. De Ponti, R. Verlato, E. Bertaglia et al., "Treatment of macro-re-entrant atrial tachycardia based on electroanatomic mapping: identification and ablation of the mid-diastolic isthmus," Europace, vol. 9, no. 7, pp. 449-457, 2007.

[75] C. Scharf, H. Oral, A. Chugh et al., "Acute effects of left atrial radiofrequency ablation on atrial fibrillation," Journal of Cardiovascular Electrophysiology, vol. 15, no. 5, pp. 515$521,2004$.
[76] K. Ellenbogen, B. S. Stambler, and M. A. Wood, "Atrial tachycardia," in Cardiac Electrophysiology. From Cell to Bedside, D. P. Zipes and F. Jalife, Eds., pp. 589-603, Saunders Elsevier, Philadelphia, Pa, USA, 5th edition, 2009.

[77] S. M. Markowitz, D. Nemirovksy, K. M. Stein et al., "Adenosine-insensitive focal atrial tachycardia. Evidence for de novo micro-re-entry in the human atrium," Journal of the American College of Cardiology, vol. 49, no. 12, pp. 13241333, 2007.

[78] F. Marchlinski, D. Callans, C. Gottlieb, E. Rodriguez, R. Coyne, and D. Kleinman, "Magnetic electroanatomical mapping for ablation of focal atrial tachycardias," Pacing and Clinical Electrophysiology, vol. 21, no. 8, pp. 1621-1635, 1998.

[79] U. Mohamed, A. C. Skanes, L. J. Gula et al., "A novel pacing maneuver to localize focal atrial tachycardia," Journal of Cardiovascular Electrophysiology, vol. 18, no. 1, pp. 1-6, 2007.

[80] J. M. Kalman, P. M. Kistler, and A. L. Waldo, "Localization of focal atrial tachycardias-back to the future... when (old) electrophysiologic first principles complement sophisticated technology," Journal of Cardiovascular Electrophysiology, vol. 18, no. 1, pp. 7-8, 2007.

[81] M. Haïssaguerre, M. Hocini, P. Sanders et al., "Localized sources maintaining atrial fibrillation organized by prior ablation," Circulation, vol. 113, no. 5, pp. 616-625, 2006.

[82] F. Atienza, J. Almendral, J. Moreno et al., "Activation of inward rectifier potassium channels accelerates atrial fibrilation in humans: evidence for a reentrant mechanism," Circulation, vol. 114, no. 23, pp. 2434-2442, 2006.

[83] S.-A. Chen, C.-E. Chiang, C.-J. Yang et al., "Sustained atrial tachycardia in adult patients: electrophysiological characteristics, pharmacological response, possible mechanisms and effectos of radiofrequency ablation," Circulation, vol. 90, no. 3, pp. 1262-1278, 1994.

[84] T. Datino, L. MacLe, X.-Y. Qi et al., "Mechanism by which adenosine restores conduction in dormant canine pulmonary veins," Circulation, vol. 121, no. 8, pp. 963-972, 2010.

[85] S. Ernst, F. Ouyang, F. Löber, M. Antz, and K. H. Kuck, "Catheter-induced linear lesions in the left atrium in patients with atrial fibrillation. An electroanatomic study," Journal of the American College of Cardiology, vol. 42, no. 7, pp. 12711282, 2003.

[86] A. E. Becker, "Left atrial isthmus: anatomic aspectos relevant for linear catheter ablation procedures in humans," Journal of Cardiovascular Electrophysiology, vol. 15, pp. 809-812, 2004.

[87] T. Rostock, M. D. O’Neill, P. Sanders et al., "Characterization of conduction recovery across left atrial linear lesions in patients with paroxysmal and persistent atrial fibrillation," Journal of Cardiovascular Electrophysiology, vol. 17, no. 10, pp. 1106-1111, 2006.

[88] C. Eitel, G. Hindricks, P. Sommer et al., "Circumferential pulmonary vein isolation and linear left atrial ablation as a single-catheter technique to achieve bidirectional conduction block: the pace-and-ablate approach," Heart Rhythm, vol. 7, no. 2, pp. 157-164, 2010.

[89] T. W. Lim, C. H. Koay, R. McCall, V. A. See, D. L. Ross, and S. P. Thomas, "Atrial arrhythmias after single-ring isolation of the posterior left atrium and pulmonary veins for atrial fibrillation," Circulation: Arrhythmia and Electrophysiology, vol. 1, no. 2, pp. 120-126, 2008.

[90] J. E. Taclas, R. Nezafat, J. V. Wylie et al., "Relationship between intended sites of RF ablation and post-procedural scar in AF patients, using late gadolinium enhancement cardiovascular magnetic resonance," Heart Rhythm, vol. 7, no. 4, pp. 489-496, 2010. 
[91] M. Del Greco, A. Cristoforetti, M. Marini, and F. Ravelli, "Image fusion shows the role of incomplete ablation lines in creating a substrate for left atrial flutter occurring after atrial fibrillation ablation," Heart Rhythm, vol. 5, no. 1, pp. 163$164,2008$.

[92] B. Schumacher, W. Jung, T. Lewalter, C. Wolpert, and B. Lüderitz, "Verification of linear lesions using a noncontact multielectrode array catheter versus conventional contact mapping techniques," Journal of Cardiovascular Electrophysiology, vol. 10, no. 6, pp. 791-798, 1999.

[93] K. Yano, K. Hirao, T. Horikawa, M. Tanaka, and M. Isobe, "Electrophysiology of a gap created on the canine atrium," Journal of Interventional Cardiac Electrophysiology, vol. 17, no. 1, pp. 1-9, 2006.

[94] S. P. Thomas, E. M. Wallace, and D. L. Ross, "The effect of a residual isthmus of surviving tissue on conduction after linear ablation in atrial myocardium," Journal of Interventional Cardiac Electrophysiology, vol. 4, no. 1, pp. 273-281, 2000.

[95] S. J. Melby, A. M. Lee, A. Zierer et al., "Atrial fibrillation propagates through gaps in ablation lines: implications for ablative treatment of atrial fibrillation," Heart Rhythm, vol. 5, no. 9, pp. 1296-1301, 2008.

[96] C.-T. Tai and S.-A. Chen, "Conduction barriers of atrial flutter: relation to the anatomy," Pacing and Clinical Electrophysiology, vol. 31, no. 10, pp. 1335-1342, 2008.

[97] P. Jaïs, D. C. Shah, M. Haïssaguerre et al., "Mapping and ablation of left atrial flutters," Circulation, vol. 101, no. 25, pp. 2928-2934, 2000.

[98] H. Nagakawa, S. Hayyar, K. Matsudaira et al., "Characterization of reentrant circuit in macrorreentrant right atrial tachycardia after surgical repair of congenital heart disease. Isolated channels between scars allow "focal" ablation," Circulation, vol. 103, pp. 699-709, 2001.

[99] F. Ouyang, S. Ernst, T. Vogtmann et al., "Characterization of reentrant circuits in left atrial macrorreentrant tachycardia. Critical isthmus block can prevent atrial tachycardia recurrence," Circulation, vol. 105, no. 16, pp. 1934-1942, 2002.

[100] S. Tzeis, A. Luik, C. Jilek et al., "The modified anterior line: an alternative linear lesion in preimitral flutter," Journal of Cardiovascular Electrophysiology, vol. 21, pp. 665-670, 2010.

[101] A. Pérez-Silva, J. L. Merino, I. Valverde et al., "Ablación del flutter auricular perimitral mediante el bloqueo del istmo mitral superior," Revista Española de Cardiología, vol. 63, supplement 3, p. 41, 2010.

[102] P. Della Bella, A. Fraticelli, C. Tondo, S. Riva, G. Fassini, and C. Carbucicchio, "Atypical atrial flutter: clinical features, electrophysiological characteristics and response to radiofrequency catheter ablation," Europace, vol. 4, no. 3, pp. 241253, 2002.

[103] N. F. Marrouche, A. Natale, O. M. Wazni et al., "Electrophysiology, anatomy and results of ablation," Circulation, vol. 109, no. 20, pp. 2440-2447, 2004.

[104] F. Bogun, B. Bender, Y.-G. Li, and S. H. Hohnloser, "Ablation of atypical atrial flutter guided by the use of concealed entrainment in patients without prior cardiac surgery," Journal of Cardiovascular Electrophysiology, vol. 11, pp. 136$145,2000$.

[105] E. Delacretaz, L. I. Ganz, K. Soejima et al., "Multiple atrial macro-re-entry circuits in adults with repaired congenital heart disease: entrainment mapping combined with threedimensional electroanatomic mapping," Journal of the American College of Cardiology, vol. 37, pp. 1665-1676, 2001.

[106] A. Frustaci, C. Chimenti, F. Bellocci, E. Morgante, M. A. Russo, and A. Maseri, "Histological substrate of atrial biopsies in patients with lone atrial fibrillation," Circulation, vol. 96, no. 4, pp. 1180-1184, 1997.

[107] M. K. Stiles, B. John, C. X. Wong et al., "Paroxysmal lone atrial fibrillation is associated with an abnormal atrial substrate," Journal of the American College of Cardiology, vol. 53, no. 14, pp. 1182-1191, 2009.

[108] J. A. Cabrera, S. Y. Ho, V. Climent, and D. Sánchez-Quintana, "The architecture of the left lateral atrial wall: a particular anatomic region with implications for ablation of atrial fibrillation," European Heart Journal, vol. 29, no. 3, pp. 356$362,2008$.

[109] S. Moreno-Reviriego, J. L. Merino, I. Valverde et al., "Cicatrices en la aurícula izquierda en pacientes con fibrilación auricular: prevalencia y posible relación con el sustrato de sostenimiento arrítmico," Revista Española de Cardiología, vol. 63, supplement 3, p. 127, 2010.

[110] A. Verma, O. M. Wazni, N. F. Marrouche et al., "Pre-existent left atrial scarring in patients undergoing pulmonary vein antrum isolation: an independent predictor of procedural failure," Journal of the American College of Cardiology, vol. 45, pp. 285-292, 2005.

[111] Y. J. Lin, S. Higa, C.-T. Tai et al., "Role of the right atrial substrate in different types of atrial arrhythmias," Heart Rhythm, vol. 6, no. 5, pp. 592-598, 2009.

[112] F. J. Chorro, J. Sanchís, L. Such et al., "Acute effects of radiofrequency ablation upon atrial conduction in proximity to the lesion site," Pacing and Clinical Electrophysiology, vol. 21, pp. 659-668, 1998.

[113] L. Di Biase, D. Burkhard, P. Mohanty et al., "Left atrial appendage: an urecognized trigger site of atrial fibrillation," Circulation, vol. 122, pp. 109-118, 2010.

[114] K. R. Chun, D. Bansch, S. Ernst et al., "Pulmonary vein conduction is the major finding in patients with atrial tachyarrythmias after intraoperative Maze ablation," Journal of Cardiovascular Electrophysiology, vol. 18, pp. 358-363, 2007.

[115] R. W. Sy, L. J. Gula, P. Leong-Sit et al., "Complete antral encirclement is not required for pulmonary vein isolation," Heart Rhythm, vol. 8, no. 1, pp. 16-22, 2011.

[116] O. Wazni, N. F. Marrouche, D. O. Martin et al., "Randomized study comparing combined pulmonary vein-left atrial junction disconnection and cavotricuspid isthmus ablation versus pulmonary vein-left atrial junction disconnection alone in patients presenting with typical atrial flutter and atrial fibrillation," Circulation, vol. 108, no. 20, pp. 24792483, 2003.

[117] A. L. Waldo and G. K. Feld, "AInter-relationships of atrial fibrillation and atrial flutter," Journal of the American College of Cardiology, vol. 51, pp. 779-786, 2008.

[118] Y. Gong, F. Xie, K. M. Stein et al., "Mechanism underlying initiation of paroxysmal atrial flutter/atrial fibrillation by ectopic foci. A simulation study," Circulation, vol. 115, no. 16, pp. 2094-2102, 2007.

[119] Y. Khaykin, A. Skanes, J. Champagne et al., "A randomized controlled trial of the efficacy and safety of electroanatomic circumferential pulmonary vein ablation supplemented by ablation of complex fractionated atrial electrograms versus potential-guided pulmonary vein antrum isolation guided by intracardiac ultrasound," Circulation: Arrhythmia and Electrophysiology, vol. 2, pp. 481-487, 2009.

[120] Y. J. Lin, H. M. Tsao, S. L. Chang et al., "The distance between the vein and lessions predicts the requirement of carina ablation in circumferential pulmonary vein isolation," Europace, vol. 13, pp. 376-382, 2011. 
[121] L. Di Biase, S. Conti, P. Mohanty et al., "General anesthesia reduces the prevalence of pulmonary vein reconnection during repeat ablation when compares with conscious sedation: results from a randomized study," Heart Rhythm Journal, vol. 8, pp. 368-372, 2011.

[122] C. Pappone, G. Vicedomini, F. Manguso et al., "Robotic magnetic navigation for atrial fibrillation ablation," Journal of the American College of Cardiology, vol. 47, no. 7, pp. 13901400, 2006.

[123] E. S. Gang, J. Merino, and B. L. Nguyen, "Remote navigation for ablation procedures," European Cardiology, vol. 6, pp. 5056, 2010.

[124] C. S. Elayi, L. Di Biase, C. Barrett et al., "Atrial fibrillation termination as a procedural endpoint during ablation in long-standing persistent atrial fibrillation," Heart Rhythm, vol. 7, no. 9, pp. 1216-1223, 2010.

[125] C. Schmit, H. Estner, B. Hecher et al., "Radiofrequency ablation o complex fractionated atrial electrograms (CFAE): preferential sites of acute termination and regularization in paroxysmal and persistent atrial fibrillation," Journal of Cardiovascular Electrophysiology, vol. 18, pp. 1039-1046, 2007. 


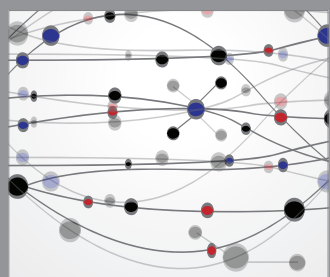

The Scientific World Journal
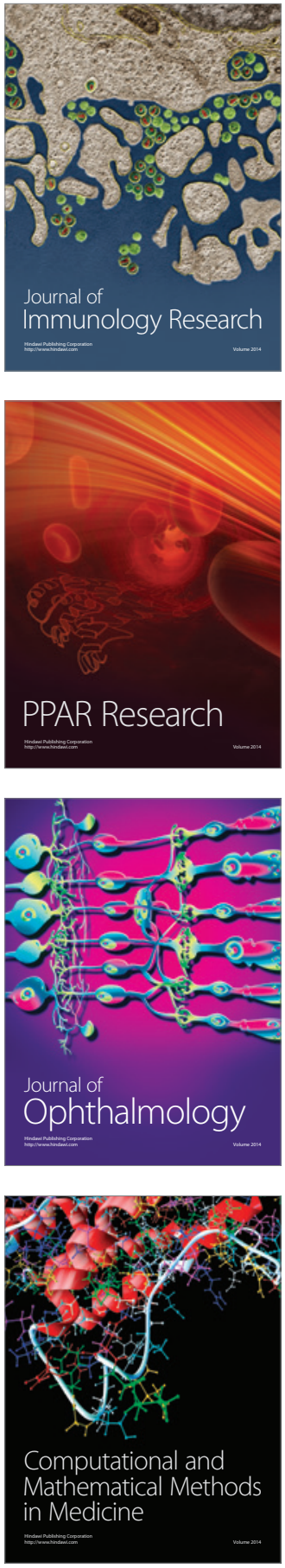

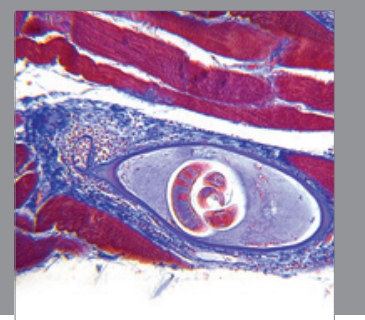

Gastroenterology

Research and Practice
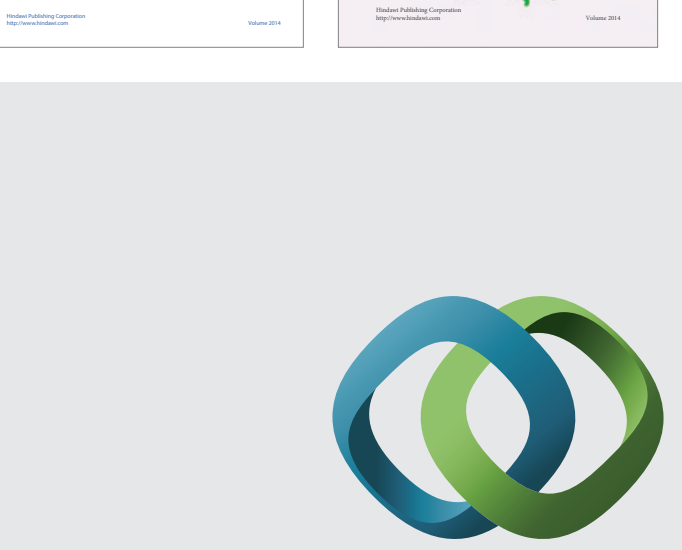

\section{Hindawi}

Submit your manuscripts at

http://www.hindawi.com
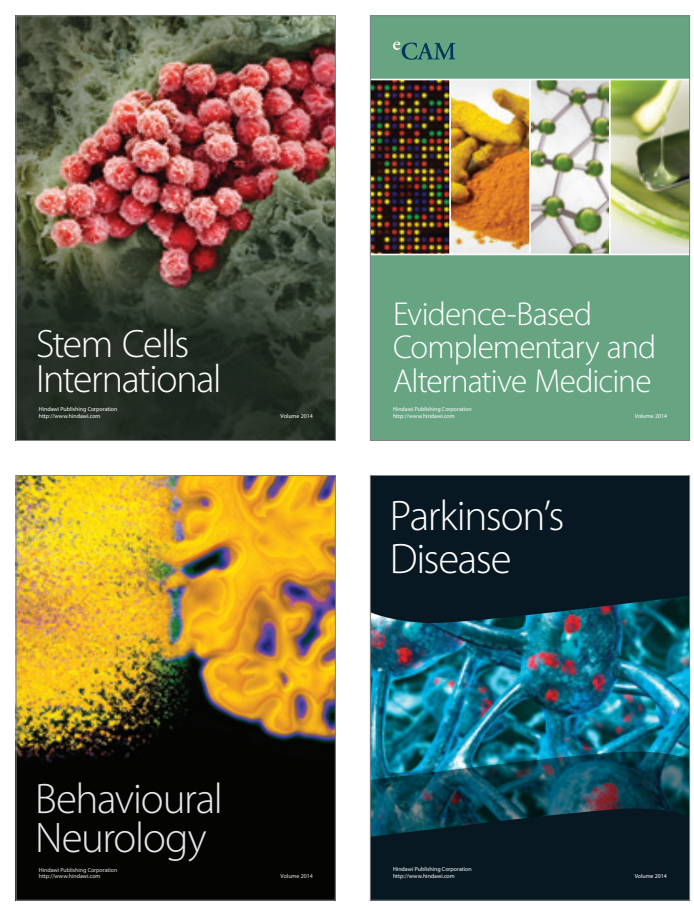

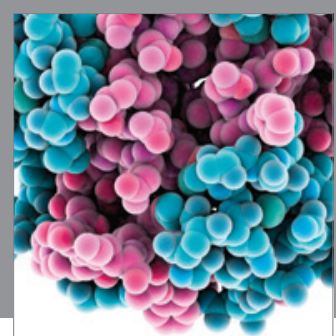

Journal of
Diabetes Research

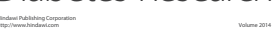

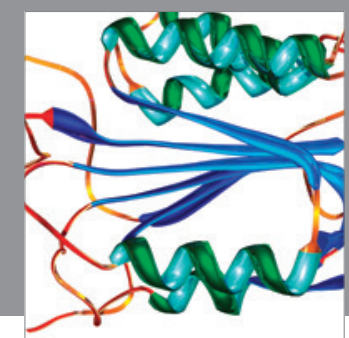

Disease Markers
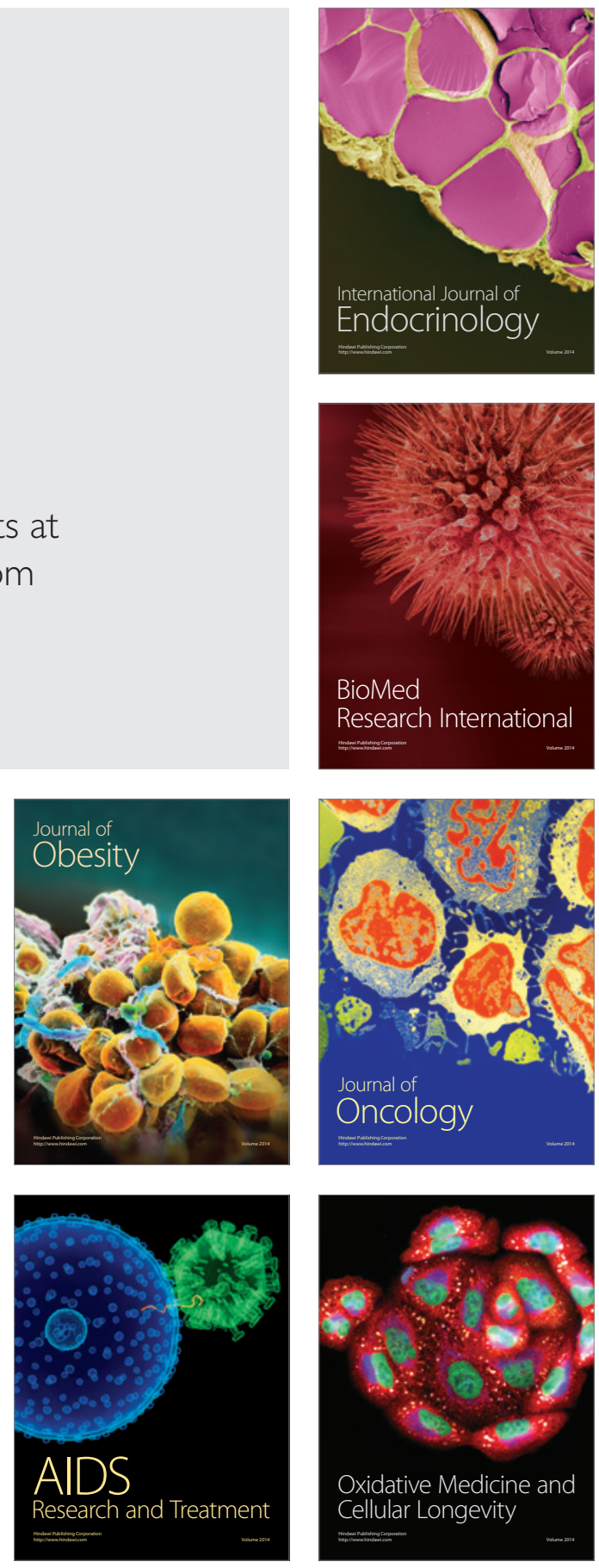